\title{
The application of teleradiology in dentomaxillofacial radiology
}

\author{
Fahmi Oscandar, Yurika A. Lita," Farina Pramanik
}

\section{Abstract}

Objective: This review is to provide information to general dentist and other dental specialist about the advantage of teleradiology in dentomaxillofacial radiology as a means of communication between dental radiologist and other dental specialists using teleradiology system.

Methods: Radiograph imaging can be easily sent from dental radiologist to other dental specialists not only in the sections of the hospital but also in other locations throughout the world.
Results: The teleradiology system needs adequate internet capacity, internet speed and band width. The benefit of using teleradiology is its ability to achieve effective dentomaxillofacial radiology services.

Conclusion: Providing radiograph interpretation services thus can provide services to the patient effectively and efficiently, without having any problem due to the human resources, time, distance and location.
Departement of Dentomaxillofacial Radiology, Faculty of Dentistry, Padjadjaran University, Bandung, Indonesia
${ }^{*}$ Correspondence to: Yurika Ambar Lita, Departement of Dentomaxillofacial Radiology, Faculty of Dentistry, Padjadjaran University, Bandung, Indonesia yurika.lita@unpad.ac.id

Received: 29 January 2016

Revised: 25 April 2016

Accepted: 26 April 2016

Available Online: 30 April 2016

Keywords: Teleradiology, Digital, Radiograph, Interpretation

Cite this Article: Oscandar F, Lita YA, Pramanik F. 2016. The Application of Teleradiology in Dentomaxillofacial Radiology. Journal of Dentomaxillofacial Science 1(1): 67-72. D0I:10.15562/jdmfs.v1i1.30

\section{Introduction}

The dentomaxillofacial radiology service in Indonesia is growing in line with technological developments. There are still problems that disrupt dentomaxillofacial radiology services, particularly in terms of location, time, limitations of $\mathrm{x}$-rays machine and human resources. In secluded areas radiology service is hard to find especially out of working hours whereas in emergency situation, the dentomaxillofacial radiology services is necessary to perform by a dentomaxillofacial radiologist. Quantity of dentomaxillofacial radiologist in Indonesia is very limited whereas it is a required demand of all dentomaxilloradiograph services.

Along with the development of science and technology, nowadays, various problems are included distance, time and human resources limit can be solved by communication device. Access to remote areas in the field of dentomaxillofacial radiology can be done by a communication device called "The teleradiology".

The teleradiology systems use once known for their digital imaging comes along with the development of telecommunication technology, which began with advancement of internet. Nowadays, the imaging can be sent, not only within the hospital but also outside the hospital even to other locations around the world. Teleradiology system has been widely used in the medical field which has also been now getting more advanced to mobile phone interpretation without any distance limit. ${ }^{1}$
The aim of this paper is to give information to dentists about the use of teleradiology in the field of dentistry to be used as a means of communication to access remote area between dentomaxillofacial radiologists and colleagues using teleradiology system.

\section{Methods}

The teleradiology is the practice of radiology through remote transmission and displaying the results of diagnostic radiographic imaging of examination of the patient. The dentomaxillofacial radiologist interpretation is prepared to transmit imaging from the place where the imaging was taken to any different location. The dentomaxillofacial radiologist can either send the radiographic image or provide written interpretation. Teleradiology practices include conventional procedures of covering intra oral and extra oral radiography, computed tomography (CT), ultrasound (US), magnetic resonance imaging (MRI) and nuclear medicine (NM). ${ }^{9}$

The interpretation of radiographic imaging by teleradiology can be performed with minimal time and provide access to a lot of consultation. Teleradiology techniques may also support the development of the continuing education in the field of radiology. The advantages of this technique are that teleradiology users in different locations can simultaneously view the image being sent. If the teleradiology can function properly, it can increase 
access to the use of radiological interpretation done, accordingly it can improve the services to patients significantly. The teleradiology system is less useful if the image sent does not provide adequate imaging quality. ${ }^{2}$

Teleradiology use was proposed to reach these advantages below:

A. To provide consultative and interpretative radiology services.

B. To make a radiology consultation that is available at medical facilities without the support of a dentomaxillofacial radiologist in place.

C. Providing timely services to radiological imaging and interpretation in clinical care.

D. To facilitate the interpretation of radiology in situations of on-call.

E. To provide subspecialty radiology support when it is needed.

F. To improve the continuing education for a dentomaxillofacial radiologist.

G. Promoting efficiency and improving quality of service.

$\mathrm{H}$. To provide imaging interpretation for referral.

I. Supporting telemedicine.

J. Providing quality surveillance imaging.

Some terms that need to be understood in teleradiology is DICOM and PACS. DICOM (Digital Imaging and Communication In Medicine) is a standard radiological imaging processing of imaging and communication of medical information between computers. ${ }^{3}$ PACS (Picture Archiving and Communication System) is imaging without films made by the method of computerized communication and data store medical imaging such as computed radiographic, digital radiographic, computed tomographic, ultrasound, fluoroscopic, magnetic resonance and $\mathrm{x}$-ray radiograph. ${ }^{4}$

The teleradiology was first published in New York Times article 1907 that describes telephotograph idea. The first experiments performed by two dental radiographs were shipped from the USA with the telegraph in 1929. The early generation of clinical teleradiology use analog TV transmissions and video ${ }^{5}$ technology made in late 1950 and continued in 1980 where teleradiology started using computer.

There are $75 \%$ dentomaxillofacial radiologists using teleradiology in group practice, 30\% in private practice and $92 \%$ the radiological practice using on a call teleradiology to provide interpretation services. Utilization of teleradiology in conventional radiography was only by $43 \%$ and magnetic resonance imaging $47 \%$. Another survey conducted by Saketkhoo et al. ${ }^{12}$ held in 114 private hospitals. It was found that 97 public hospitals $(82 \%)$ use teleradiology at night time. The mentioned data may indicate that the dentomaxillofacial radiologist is greatly benefited for the application of teleradiology which is trust worthy, but still there should be a critical requirement to concern in matters of accuracy and appropriate services time. ${ }^{6}$

The principles of teleradiology components required in teleradiology system. ${ }^{5}$

\section{A. Image Sending Station}

At the image sending center, radiographs captured in digital format by the digitizer, if initially in the form of x-ray films using a film scanner CCD (charge coupled device). Today, most of the image is already in digital format DICOM (digital imaging and communication in medicine), which is a direct result of digital modalities. ${ }^{3,5}$

\section{B. Transmission Networks}

The communications network is essential for teleradiology. Networks can be either wired or wireless. The simplest cable network is a telephone line or ISDN (integrated services digital network) digital that can be used as a connection point to another with the help of a modem that is known as a dial-up connection, because they appear if required. This is less stringent in security requirements, because there is no other partner in the communication path. The speed that can be used for modem connections is 9.6 $\mathrm{kbit} / \mathrm{s}$ and $64 \mathrm{kbit} / \mathrm{s}$ for the ISDN connection path. Integrated services digital network connections can be duplicated: using 2-6 parallel channels with a speed of 128-384 kbits/s. ${ }^{3-5}$ Connections Wide area network (WAN) is a fixed connection between the local area network (LAN) at different installations, providing higher speeds and reliable connections. Solutions with lower costs for the WAN connection are using DSL (Digital Subscriber Line) connection at the customer site. One of the WAN connections is the Internet, but includes the possibility for an easier relationship, even on an ad hoc basis. Wireless network connection to teleradiology including satellite transmission, microwave transmission, and mobile phone connections. Satellite connections are used directly or as part of a WAN connection. Microwave transmission usually involves the point-to-point direct connection. Phone's network connection is based on the ability to transfer data from the line operator and is usually connected to the LAN at the delivery location. ${ }^{5}$

\section{Review station}

In a review of teleradiology systems, high-end monitor with resolution of $1024 \times 1024$ pixel $(1 \mathrm{~K})$ is recommended for cross-sectional images and forprimary diagnosis digital radiography resolution 


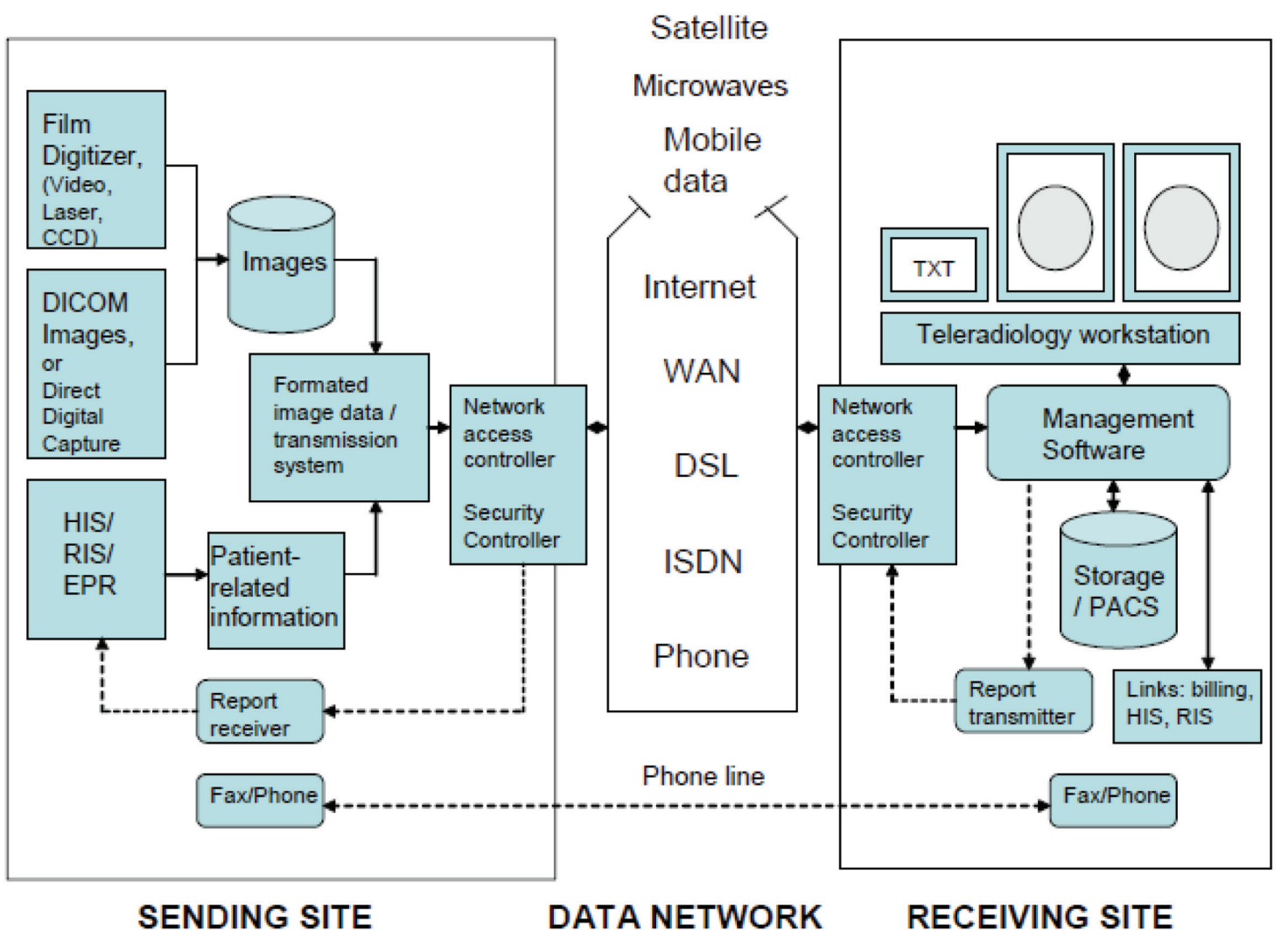

Figure 1 Teleradiology Component ${ }^{8,7}$

should include local storage for images and software that is user-friendly to manage and display image sand relevant patient data. The teleradiology work-station should be connected the local PACS forstoring images and image capture, administration tools for reporting and billing is also required. ${ }^{5}$

The electronic radiographic imaging of patient is done by creating a code in digital format on the imaging dispatch center sent over transmission lines, received, displayed and stored in the reception center imaging.

Web-based teleradiology system with a web browser is commonly found in personal computers, but a software is needed to display the advanced digital image. The ability for all types of computers is to display the image in a way that is easy, safe and reliable and it is important to distribute the image to users. Workstations are necessary but require a relatively expensive cost that is only possible in the radiology department of dentistry, which has a substantial funding.

Compression imaging, internet connection, and a standard document format is very useful to achieve that goal. Three concepts can be implemented together with a solution for imaging consultation remotely without requiring special software to display DICOM. The appropriate solu- tion is applied to a secluded place where broadband connections are less adequate. ${ }^{8}$ The work flow of teleradiology in general are the referral from dentist will send all the to information into an online form with your dentomaxillofacial radiologist who has competence as teleradiologist, The dentomaxillofacial radiologist receives consul and sends to the operator radiologist (in the radiology department of dentistry), Operator radiologist in accordance with the referral is received, the results are sent to dentomaxillofacial radiologist to do interpretation and the dentomaxillofacial radiologist sends the results interpretation online. ${ }^{14}$

Radiological examination in the hospital should be done at least by expertly trained operator. All components in this case the operator must be licensed or registered radiology. Operators should be under the supervision of a licensed physician and have a qualified medical physicist or expert in imaging management. Interpretation of imaging should be performed by physicians who have an understanding of the basic teleradiology technology, which covers aspects of the strengths and weak nesses (limitations) and who is knowledgeable 
in the use of teleradiology equipment and be able to demonstrate appropriate qualification standards for diagnostics by teleradiology. Radiology operator, radiation therapists, nuclear medicine operator or sonogram operator must be certified or state licensed and trained to operate and supervise the teleradiological system. ${ }^{1,13}$

Teleradiology has advantage in patient care. The potential advantage of teleradiology is the process of evaluating patients in emergency room and the use of teleradiology can reduce the distance from home to the hospital and can be used to serve the needs of radiography and its interpretation of the different locations. ${ }^{12}$ It can be a strategy for dentomaxillofacial radiologist in overcoming the limited amount of resources and distance factors in an effort to meet the needs of the practice of radiology services in the field of dentistry. Potential negative factor of teleradiology was the rising cost in this service. Because it requires a fairly expensive digital device in preparing the software and hardware to provide a good service. ${ }^{10}$

Some of the benefits of teleradiology $\operatorname{are}^{8}$

A. Teleradiology create a community of patients who are in distant places with limited visits to a dentomaxillofacial radiologist is easy to obtain radiographic imaging results and send it back. ${ }^{4}$ This makes more efficient use of teleradiology system from economic

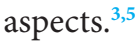

B. Imaging with complex problems can be transmitted from the hospital to do service to other hospitals. This is advantageous because hospital patients are more capable to decide whether or not a patient is required to be transferred from smaller hospitals to the large hospital without disturbing the comfort of patient. It also said that the use of teleradiology system leads to a reduction of $50 \%$ and in the case of patient transport is not necessary. ${ }^{3,5}$

C. The use of teleradiology can be used as an efficient method to generate reports quickly during emergencies where there is no dentomaxillofacial radiologist at the hospital during that study. ${ }^{4}$ Fernandez Salazar reported that teleradiology can reduce delays in care after doing diagnostic., ${ }^{3,5}$

D. Allowing teleradiology into educational media presentations and tutorials in the radiology department at the hospital. ${ }^{4}$

E. It can display images in different locations simultaneously for the discussion purpose. ${ }^{4}$

\section{Results}

Teleradiology is a big business in the United state where there are many private teleradiology companies that employs radiologists to provide teleradiology interpretation. Upon surveying a few years ago showed that the majority of radiology practices were using teleradiology services. ${ }^{10}$

\section{Deficiencies in the diagnostic accuracy}

Since the teleradiology system was introduced, there exists one aspect of concern due to the accuracy of diagnostics. Telediagnostics has more accurate condyle fractures imaging compared to direct visualization where the fractures of midfacial transmitted through the film showed the simple quality, further the accuracy of diagnosis was also lower than indirect vision. ${ }^{11}$

\section{Confidentiality of data}

Teleradiology services running on the website, at the time of upload imagery contained personal information of patients. Medical records of the patient's personal information that are transmitted using teleradiology systems must be kept secret. Therefore, teleradiology system users in the service must be educated about the confidentiality of the data security information. ${ }^{3}$ A method to protect data from unauthorized access to patient's privacy. ${ }^{9}$ It also calls for the protection of data and guarantee data integrity to prevent damage to data intentionally or not.'

Studies in teleradiology have focused on three main objectives:

A. To reduce the amount of memory required to encode imaging radiography, keeping information, improvement of digital imaging and communications in medical.

B. Increase rate of transmission on the network, although the distribution of broadband internet connections in western countries is very good but in some areas other than western countries is less than optimal.

C. Fix the display of digital imaging and communication in medical field.?

\section{Discussion}

Dentomaxillofacial radiology science in Indonesia had developed rapidly in recent years. Consulting services of dentomaxillofacial radiology interpretation should be efficient and effective to supported diagnostic and treatment plan in dentistry. There are common obstacles that arise in Indonesia:

A. During the processing and radiographic interpretation, patients should be waiting because of the use of conventional system, processing dental film and delivery of 
radiographic films between departments with travelling distances needed.

B. Another obstacle with consultancy services for radiographic interpretation is the distance the services cannot reach the remote areas where the internet access is not available.

C. One of the biggest obstacles is the limited ratio of dentomaxillofacial radiologist compared to quantity of service needed.

Increase of necessity for efficient and effective consulting service and dental radiology interpretation for supporting diagnostic and treatment plan. Various obstacles lead to unfulfilled demand of efficient and effective consulting services and dental radiology interpretation.

Among the developmental of digital imaging technology in medicine, digital imaging becomes a necessity nowadays for diagnosis and treatment planning. Teleradiology is used as a media of communication in the field of dentomaxillofacial radiology especially to provide effective patient services, without the issue of limited human resources, time, distance and location.

Because of insufficient quantity of dentomaxillofacial radiologist throughout Indonesia, alternative techniques are required in the field of remote communication to supply the needs of dentomaxillofacial radiography services of any hospitals spreading all over Indonesia. To resolve this obstacles require a comprehensive system. The development of technology helps to resolve drawbacks like: the long distance, time and human resources by long-distance communication.

Communication at distance using electronic transmission in the field of radiology is known as teleradiology. Teleradiology systems provide services to patients more efficiently, especially for nighttime emergency room or hospital in a remote area with limited dentomaxillofacial radiologist. Dental imaging can be easily sent not only within the hospital but also outside the hospital and to other locations around the world.

The existence of this teleradiology system can resolve the obstacles, but there is still less application of teleradiology in the field of dentistry. This discussion is intended to introduce teleradiology system in the field of dentistry in Indonesia which is expected to be applied and further developed in the field of dentistry in Indonesia.

Developments in technology informatics lead to the rapid development of teleradiology. The electronic transmission of radiographic imaging from one location to another for various purposes, especially for radiograph interpretation, consultation and research. ${ }^{6,7,11}$ Users in different locations can view simultaneously radiograph image. Utilization of teleradiology appropriately can increase access to radiograph interpretation which also improve the services to patients. $^{10}$

The supporting key factor of teleradiology systems is the changing paradigm about processing and using of radiograph imaging. Since the discovery of X-rays by Roentgen in 1895, the imaging process since hundreds of years ago was based on the chemical photograph and physics of imaging. Modern appliances such as the Nuclear Medicine and Computed Tomographic imaging is displayed and stored digitally using a computer in 1960 and 1971 which was the beginning of the migration from conventional into digital imaging. The positive impact of the use of digital imaging and teleradiology can also reduce the use of compact disc, chemical waste use than conventional radiograph.

\section{Conclusion}

Teleradiology can be used as communication media between dentomaxillofacial radiologists with other dental specialists, especially in providing radiograph interpretation services thus can provide services to patient effectively and efficiently, without problem of human resources, time, distance and location.

\section{References}

1. Choi JW. Clinical usefulness of teleradiology in general dental practice. Imaging Sci Dent 2013;43: 99-104.

2. American college of Radiology. ACR Standard for teleradiology. 13-21. Available at: www.acr.org.

3. Huang HK. PACS and imaging informatics basic principles and applications. Hoboken: John Wilet \& Sons, Inc; 2004.

4. Tong-Carrison KS, Wong E. Governance of picture archiving and communication systems: Data security and quality management of filmless radiology. Hershey: Information Science Reference; 2009.

5. Reponen J. Teleradiology Changing Radiological Service Process From local to regional, international and mobile environment. ACTA Universitas Oulu 2010.

6. Larson DB, Cypel YS, Forman HP, et al. A comprehensive portrait of teleradiology in radiology practices: results from the American College of Radiology's 1999 survey. AJR Am J Roentgenol 2005; 185: 24-35.

7. Kalyanpur A, Neklesa VP, Pham DT, et al. Implementation of an international teleradiology staffing model. RSNA 2004;232: 415-419.

8. European society of radiology. Teleradiology I. 2004. Available at: www.myESR.org.

9. Hunter TB, Krupinski EA. University-based teleradiology in the United States. Journal of Healthcare.2014, 2, 192206; doi: 10. 3390/healthcare2020192 available at: www. mdpi.com/journal/healthcare.

10. Jelic D, Dragutinovic G. Development of teleradiology in private practice in Serbia and its influence on increased efficiency and effectiveness of medical professionals. J Theory \& Practice Management 2013;69: 85-91. 
11. Luccichenti G, Cademartiri F, Pichiecchio A, et al. User inteface of teleradiology system for MR assessment of multiple sclerosis. J Digital Imaging 2009.

12. Saketkhoo DD, Bhargavan M, Sunshine JH, et al. Emergency department image interpretation services at private community hospitals. Radiology 2004;231: 190-197.

13. Silva E, Breslau J, Barr RM, et al. ACR white paper on teleradiology practice: A report from the task force on teleradiology practice. J Am Coll Radiol 2013;10: 575-585.
14. Martinon A, Berthezene Y, Thomson V, et al. Evaluating the impact of teleradiology on emergency care in Lyon France. EPOS 2012;1606: 1-22.

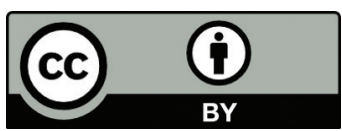

This work is licensed under a Creative Commons Attribution 